\title{
INERTIAL MANIFOLD FOR A REACTION DIFFUSION EQUATION MODEL OF COMPETITION IN A CHEMOSTAT
}

\author{
JOSEPH WAI HUNG SO ${ }^{1,2}$
}

(Received 6 March 1990; revised 18 June 1990)

\begin{abstract}
The existence of an inertial manifold for a reaction-diffusion equation model of the chemostat is established.
\end{abstract}

\section{Introduction}

The purpose of this paper is to show that inertial manifolds exist for a system of reaction diffusion equations which was used to model competition in a chemostat (c.f. So and Waltman [8]). The equations are:

$$
\left\{\begin{array}{l}
S_{t}=S_{x x}-f(S) u-g(S) v \\
u_{t}=u_{x x}+f(S) u \\
v_{t}=v_{x x}+g(S) v
\end{array}\right.
$$

where $S(t, x)$ (respectively $u(t, x), v(t, x)$ ) denotes the concentration of the limiting substrate (respectively the competing micro-organisms) at time $t \geq 0$ and position $0 \leq x \leq L$. Here

$$
\left\{\begin{array}{l}
f(S):=m S /(a+S) \\
g(S):=n S /(b+S)
\end{array}\right.
$$

\footnotetext{
${ }^{1}$ International Institute for Applied System Analysis, Laxenburg, Austria.

${ }^{2}$ Permanent address: Department of Mathematics, University of Alberta, Edmonton, Canada T6G 2 G1.

(C) Copyright Australian Mathematical Society 1991, Serial-fee code 0334-2700/91
} 
for $S \geq 0$, where $m, a, n$ and $b>0$. The boundary conditions are

$$
\left\{\begin{array}{l}
S_{x}(t, 0)=-S^{(0)} \\
u(t, 0)=v_{x}(t, 0)=0 \\
S(t, L)+\gamma S(t, L)=u_{x}(t, L)+\gamma u(t, L)=v_{x}(t, L)+\gamma v(t, L)=0
\end{array}\right.
$$

where $S^{(0)}$ and $\gamma>0$.

Let $z=S+u+v$. Then $z$ satisfies

$$
z_{t}=z_{x x}
$$

with boundary conditions

$$
\left\{\begin{array}{l}
z_{x}(t, 0)=-S^{(0)} \\
z_{x}(t, L)+\gamma z(t, L)=0 .
\end{array}\right.
$$

We need the following form of the Poincare inequality.

Proposition 1.1. (c.f. Theorem 11.11 of Smoller [7]). Let $w \epsilon W^{1,2}[0, L]$. Then

$$
\left\|w^{\prime}\right\|_{2}^{2}+\gamma w(L)^{2} \geq c\|w\|_{2}^{2},
$$

where $c>0$ is the smallest eigenvalue of the boundary-value problem

$$
-w^{\prime \prime}=\lambda w, \quad w^{\prime}(0)=w^{\prime}(L)+\gamma w(L)=0 .
$$

Proposition 1.2. Let $z(t, x)$ be a solution of (1.4) and (1.5). Then $z(t, x)$ converges to the steady state solution $\hat{z}(x):=S^{(0)}(L+1 / \gamma-x)$ of $(1.4),(1.5)$ in the $L^{2}$ norm.

Proof. Let $w=z-\hat{z}$. Then $w$ satisfies $w_{t}=w_{x x}$ and $w_{x}(t, 0)=$ $w_{x}(t, L)+\gamma w(t, L)=0$. Now

$$
\begin{aligned}
\frac{1}{2} \frac{d}{d t}\left(\int_{0}^{L} w^{2} d x\right) & =\int_{0}^{L} w \frac{d w}{d t} d x=\int_{0}^{L} w w_{x x} d x \\
& =\left[w w_{x}\right]_{0}^{L}-\int_{0}^{L} w_{x}^{2} d x=-\gamma w(t, L)^{2}-\int_{0}^{L} w_{x}^{2} d x
\end{aligned}
$$

By Proposition (1.1),

$$
\frac{1}{2} \frac{d}{d t}\|w(t, .)\|_{2}^{2} \leq-c\|w(t, .)\|_{2}^{2}
$$

which in turn implies

$$
\|w(t, .)\|_{2} \leq e^{-c t}\|w(0, .)\|_{2}
$$


Since we are only interested in asymptotic behavior, we replace $\hat{z}(x)$ in (1.1) and (1.3) to obtain

$$
\left\{\begin{array}{l}
u_{t}=u_{x x}+f(\hat{z}(x)-|u|-|v|) u \\
v_{t}=v_{x x}+g(\hat{z}(x)-|u|-|v|) v
\end{array}\right.
$$

with boundary conditions:

$$
u_{x}(t, 0)=v_{x}(t, 0)=u_{x}(t, L)+\gamma u(t, L)=v_{x}(t, L)+\gamma v(t, L)=0,
$$

where

$$
\begin{aligned}
& f(S):= \begin{cases}m S /(a+|S|) & \text { for } S \geq-1 \\
-m /(a+1) & \text { for } S<-1\end{cases} \\
& g(S):= \begin{cases}n S /(b+|S|) & \text { for } S \geq-1 \\
-n /(b+1) & \text { for } S<-1\end{cases}
\end{aligned}
$$

Note that this re-definition of $f(S)$ and $g(S)$ will not affect solutions $(S(t, x), u(t, x), v(t, x))$ of $(1.1),(1.3)$ satisfying $S(t, x), u(t, x), v(t, x)$ $\geq 0$ and $S(t, x)+u(t, x)+v(t, x)=\hat{z}(x)$. It is (1.8), (1.9) for which we shall show that inertial manifolds exist.

We shall need the following simple estimates on $f$ and $g$.

Proposition 1.3. For all $S, S_{1}$ and $S_{2}$, we have

$$
\begin{aligned}
|f(S)| & \leq m, \quad|g(S)| \leq n, \\
\left|f\left(S_{1}\right)-f\left(S_{2}\right)\right| & \leq(m / a)\left|S_{1}-S_{2}\right|, \quad\left|g\left(S_{1}\right)-g\left(S_{2}\right)\right| \leq(n / b)\left|S_{1}-S_{2}\right| .
\end{aligned}
$$

\section{Inertial manifolds: general theory}

There are a number of existence theories for inertial manifolds (e.g. Kamaev [4], Mora [6], Foias, Sell and Teman [2], Mallet-Paret and Sell [5], Chow and $L u[1]$ and Teman [9]). In this section we recall one that is immediately applicable to (1.8) and (1.9).

Consider an abstract evolution equation of the form

$$
\frac{d w}{d t}+A w=R(w)
$$

on a Hilbert space $H . A$ is a linear, unbounded, self-adjoint operator on $H$ with dense domain, $D(A)$, in $H$. Moreover, $A$ is assumed to be positive and that $A^{-1}$ is compact. Under these assumptions on $A$, there exists an orthonormal basis $\left\{w_{j}\right\}$ of $H$ consisting of eigenvectors of $A, A w_{j}=\lambda_{j} w_{j}$, 
where the eigenvalues satisfy $0<\lambda_{1} \leq \lambda_{2} \leq \ldots, \lambda_{j} \rightarrow \infty$ as $j \rightarrow \infty$. The nonlinear term $R: H \rightarrow H$ is assumed to be locally Lipschitz continuous. Definition 2.1. A subset $M$ of $H$ is said to be an inertial manifold for (2.1) if it satisfies the following properties:

(i) $M$ is a finite dimensional Lipschitz manifold,

(ii) $M$ is positively invariant, and

(iii) $M$ attracts exponentially all solutions of (2.1).

Assume that (2.1) is dissipative, i.e., there is a $\rho_{0}>0$ such that

$$
\limsup _{t \rightarrow \infty}\|w(t)\|_{2} \leq \rho_{0},
$$

for all solutions $w(t)$ of (2.1). In this case, one can modify (2.1) to the so-called prepared equation

$$
\frac{d w}{d t}+A w=\theta_{\rho}(|w|) R(w)
$$

Here, $\theta:[0, \infty) \rightarrow[0,1]$ is a fixed smooth function with $\theta(s)=1$ for $0 \leq s \leq 1, \theta(s)=0$ for $s \geq 2$ and $\left|\theta^{\prime}(s)\right| \leq 2$ for $s \geq 0$. And $\theta_{\rho}(s)=\theta\left(\frac{s}{\rho}\right)$ for $s \geq 0$, where $\rho=2 \rho_{0}$.

TheOREM 2.2. (Theorem 2.2 of [Foias, Sell and Teman]). Under the above assumptions, there exist $N_{0}, K_{12}, K_{13}>0$ such that if one has

$$
N \geq N_{0}, \quad \lambda_{N+1} \geq K_{12}, \quad \lambda_{N+1}-\lambda_{N} \geq K_{13},
$$

then (2.3) possesses an inertial manifold of dimension $N$.

\section{Inertial manifolds: our model}

In order to show that $(1.8),(1.9)$ possess an inertial manifold, we will first cast them in the form (2.1) and verify the hypotheses of Theorem 2.2. Let $H$ be the Hilbert space $L^{2}[0, L] \times L^{2}[0, L]$. Let $A$ be the linear operator $\left(-d^{2} / d x^{2},-d^{2} / d x^{2}\right)$ defined on the subspace of $H$ consisting of all pairs $(u, v)$, where $u, v \in C^{2}[0, L]$ satisfy the boundary conditions (1.9). By Friedrichs' extension theorem, we can extend $A$ to a closed operator, again denoted by $A$. Then $A$ is an unbounded, self-adjoint, positive operator from its domain $D(A)$ to $H$ with $A^{-1}$ compact. Moreover, if we denote the eigenvalues of $A$ by $0<\lambda_{1} \leq \lambda_{2} \leq \ldots$, then $\lambda_{2 n-1}=\lambda_{2 n}=\mu_{n}^{2}$, where $\mu_{n}$ is the $n$-th positive root of the equation $\tan (\mu L)=\gamma / \mu$. Since $(n-1) \pi L^{-1}<\mu_{n}<\left(n-\frac{1}{2}\right) \pi L^{-1},(2.4)$ can be satisfied with a large enough $N$. 
Let $R: H \rightarrow H$ denote the Nemitski operator corresponding to the reaction term, i.e.

$$
R(u, v)(x)=(f(\hat{z}(x)-|u(x)|-|v(x)|) u(x), g(\hat{z}(x)-|u(x)|-|v(x)|) v(x)) .
$$

We first show that $R$ is globally Lipschitz continuous on $H$. Consider the integral

$$
l:=\int_{0}^{L}\left|f\left(\hat{z}(x)-\left|u_{1}(x)\right|-\left|v_{1}(x)\right|\right) u_{1}(x)-f\left(\hat{z}(x)-\left|u_{2}(x)\right|-\left|v_{2}(x)\right|\right) u_{2}(x)\right|^{2} d x
$$

Then

$$
I=\int_{M_{1}^{+} \cap M_{2}^{+}}+\int_{M_{1}^{-} \cap M_{2}^{+}}+\int_{M_{1}^{+} \cap M_{2}^{-}}+\int_{M_{1}^{-} \cap M_{2}^{-}},
$$

where

$$
\begin{aligned}
& M_{i}^{+}:=\left\{x \in[0, L]: \hat{z}(x)-\left|u_{i}(x)\right|-\left|v_{i}(x)\right|>-1\right\}, \\
& M_{i}^{-}:=\left\{x \in[0, L]: \hat{z}(x)-\left|u_{i}(x)\right|-\left|v_{i}(x)\right| \leq-1\right\} .
\end{aligned}
$$

Denote these integrals by $I_{1}, I_{2}, I_{3}$ and $I_{4}$, respectively.

For $x \in M_{1}^{-} \cap M_{2}^{-}$, the absolute value (i.e. without the square) in the integrand of $I$ is (with the $x$ suppressed):

$$
\leq\left|\left(-\frac{m}{a+1} u_{1}\right)-\left(-\frac{m}{a+1} u_{2}\right)\right| \leq \frac{m}{a+1}\left|u_{1}-u_{2}\right| \text {. }
$$

Therefore, $I_{4} \leq c_{4}\left\|u_{1}-u_{2}\right\|_{2}^{2}$, for some $c_{4}>0$.

For $x \in M_{1}^{+} \cap M_{2}^{+}$, by Proposition 1.3, the absolute value is:

$$
\begin{aligned}
& \leq\left|f\left(\hat{z}-\left|u_{1}\right|-\left|v_{1}\right|\right)\left(u_{1}-u_{2}\right)\right|+\left|\left(f\left(\hat{z}-\left|u_{1}\right|-\left|v_{1}\right|\right)-f\left(\hat{z}-\left|u_{2}\right|-\left|v_{2}\right|\right)\right) u_{2}\right| \\
& \leq m\left|u_{1}-u_{2}\right|+\frac{m}{a}|| u_{1}|+| v_{1}|-| u_{2}|-| v_{2}||\left|u_{2}\right| \\
& \leq m\left|u_{1}-u_{2}\right|+\frac{m(\hat{z}(0)+1)}{a}\left(\left|u_{1}-u_{2}\right|+\left|v_{1}-v_{2}\right|\right) .
\end{aligned}
$$

Therefore, $I_{1} \leq c_{1}\left(\left\|u_{1}-u_{2}\right\|_{2}+\left\|v_{1}-v_{2}\right\|_{2}\right)^{2}$, for some $c_{1}>0$.

There are similar estimates on $I_{2}$ and $I_{3}$ as well as on the second component of $R$. Hence, $R$ is globally Lipschitz continuous.

Next we will show that the dissipative condition (2.2) is satisfied. Integrating

$$
u u_{t}=u u_{x x}+f(\hat{z}-|u|-|v|) u^{2}
$$


we get

$$
\begin{aligned}
\frac{1}{2} \frac{d}{d t} \int_{0}^{L} u^{2} & =-\gamma u(., L)^{2}-\int_{0}^{L} u_{x}^{2}+\int_{0}^{L} f(\hat{z}-|u|-|v|) u^{2} \\
& \leq-c \int_{0}^{L} u^{2}+\int_{0}^{L} f(\hat{z}-|u|-|v|) u^{2}
\end{aligned}
$$

by Proposition 1.1. Fix any $t$ and consider the integral

$$
I:=\int_{0}^{L} f(\hat{z}-|u|-|v|) u^{2}=\int_{M^{+}} f(\hat{z}-|u|-|v|) u^{2}+\int_{M^{-}} f(\hat{z}-|u|-|v|) u^{2}
$$

where

$$
\begin{aligned}
& M^{+}:=\{x \in[0, L]: \hat{z}(x)-|u(t, x)|-|v(t, x)|>-1\} \\
& M^{-}:=\{x \in[0, L]: \hat{z}(x)-|u(t, x)|-|v(t, x)| \leq-1\} .
\end{aligned}
$$

Denote these integrals by $I_{1}$ and $I_{2}$ respectively. The first integral $I_{1}$ is bounded above by

$$
m \int_{M^{+}} u^{2} \leq m(\hat{z}(0)+1)^{2} L:=K .
$$

Let $\bar{\rho}>0$ be such that $\bar{\rho}^{2}=\max \left\{\frac{(a+1) K}{m}, \frac{(b+1) K}{n}\right\}+(\hat{z}(0)+1)^{2} L$ and pick any $\rho_{0}>\bar{\rho}$. Suppose $\|u(\bar{t}, .)\|_{2} \geq \rho_{0}$ for some $\bar{t}$. Then for $t=\bar{t}$, we have

which implies

$$
\int_{\left\{\begin{array}{l}
x \in[0, L] ; \\
|u(t, x)|<z(x)+1
\end{array}\right.} u^{2}+\int_{\left\{\begin{array}{l}
x \in[0, L] ; \\
|u(t, x)| \geq z(x)+1
\end{array}\right.} u^{2} \geq \rho_{0}^{2}
$$

$$
\begin{aligned}
& \int_{\left\{\begin{array}{l}
x \in[0, L] ; \\
|u(t, x)| \geq \hat{z}(x)+1
\end{array}\right\}} u^{2} \geq \rho_{0}^{2}-\int_{\left\{\begin{array}{l}
x \in[0, L] ; \\
|u(t, x)|<\hat{z}(x)+1
\end{array}\right\}} u^{2} \\
& \geq \rho_{0}^{2}-(\hat{z}(0)+1)^{2} L>\frac{(a+1) K}{m}
\end{aligned}
$$

Therefore, at $t=t$,

$$
I_{2} \leq-\frac{m}{a+1} \int_{M^{-}} u^{2} \leq-\frac{m}{a+1} \int_{\left\{\begin{array}{l}
x \in[0, L] ; \\
|u(t, x)| \geq z(x)+1
\end{array}\right\}} u^{2}<-K .
$$

Hence, $I<0$ and consequently $\frac{d}{d t}\|u(t, .)\|^{2} \leq-2 c\|u(t, .)\|^{2}$, whenever $\|u(t, .)\|^{2} \geq \rho_{0}$. Similarly, $\frac{d}{d t}\|v(t, .)\|^{2} \leq-2 c\|v(t, .)\|^{2}$, whenever $\|v(t, .)\|^{2}$ $\geq \rho_{0}$.

Thus, by Theorem 2.2, we have proved that the prepared equation for (1.8), (1.9) possesses an inertial manifold $M_{\rho_{0}}$.

Actually the above argument shows a little more. If we let

$$
B:=\left\{(u, v) \in H:\|u\|_{2},\|v\|_{2}<\rho_{1}\right\},
$$


where $\rho_{1}>\bar{\rho}$ then $B$ is positively invariant and absorbing, i.e., if we denote the solution operator for (1.8), (1.9) by $T(t)$ then $T(t) B \subset B$ and for each bounded set $B_{1}$, there exists $t_{1}$ such that $T(t) B_{1} \subset B$ for all $t \geq t_{1}$. Moreover, $T(t)$ maps bounded sets to bounded sets. Hence, by Theorem 4.2.4 of Hale [3], (1.8), (1.9) possess a global attractor which lies in $B$. If we now pick $\rho_{0}>\rho_{1}$ so large that the ball in $H$ with radius $\rho_{0}$ and centered at the origin contains the $B$, then $B \cap M_{\rho_{0}}$ is an inertial manifold for (1.8), (1.9). Thus, we have proved that

THEOREM 3.1. Under the above assumptions, (1.8), (1.9) possess an inertial manifold.

\section{Acknowledgements}

The author would like to thank Karl Sigmund and Josef Hofbauer for their invitation to visit Austria where this research was carried out. He would also like to thank Josef Hofbauer and Paul Waltman for many useful comments on this paper. This research was supported in part by grants from the Natural Sciences and Engineering Research Council of Canada, the Central Research Fund of the University of Alberta and Fonds zur Förderung für wissenschaftliche Forschung of Austria.

\section{References}

[1] S.-N. Chow and K. Lu, "Invariant manifolds for flows in Banach spaces", J. Diff. Equ. 74 (1988) 285-317.

[2] C. Foias, G. R. Sell and R. Teman, "Inertial manifolds for nonlinear evolutionary equations", J. Diff. Equ. 73 (1988) 309-352.

[3] J. K. Hale, Asymptotic behavior of dissipative systems, Mathematical Survey and Monographs 25, Amer. Math. Soc., Providence, 1988.

[4] D. A. Kamaev, “Hopf's conjecture for a class of chemical kinetics equations”, J. Soviet Math. 25 (1984) 836-849.

[5] J. Mallet-Paret and G. R. Sell, "Inertial manifolds for reaction diffusion equations in higher space dimensions", J. Amer. Math. Soc. 1 (1988) 805-866.

[6] X. Mora, "Finite dimensional attracting manifolds in reaction-diffusion equations", Contemporary Math. 17 (1983) 353-360.

[7] J. Smoller, Shock waves and reaction-diffusion equations, Springer-Verlag, New York, 1983.

[8] J. W.-H. So and P. Waltman, "A nonlinear boundary value problem arising from competition in the chemostat", Appl. Math. Comp. 32 (1989) 169-183.

[9] R. Teman, Infinite dimensional dynamical systems in mechanics and physics, Springer-Verlag, New York, 1988. 